\title{
Subsurface Defect Evaluation in Aluminum using Low-Frequency Thermal Images
}

\author{
Yoonjae Chung, Ranjit Shrestha and Wontae Kim ${ }^{\dagger}$ \\ Department of Mechanical \& Automotive Engineering, Kongju National University, 1223-24 \\ Cheonan-daero, Seobuk-gu, Cheonan-si, Chungcheongnam-do, 31080, South Korea \\ ${ }^{\dagger}$ Corresponding author. E-mail address: kwt@kongju.ac.kr
}

\begin{abstract}
:
In these days, as the demand for rapid and effective maintenance and condition diagnosis technology is increasing in industrial facilities, it is necessary to develop the nondestructive inspection technology of aluminum structures used in various industrial fields such as air, ship, vehicle, and power plant piping. In this study, lock-in thermography technique was applied to evaluate defect which have different sizes and depth. Aluminum plate specimens excited different frequency with periodic signal. It is obtained phase and amplitude images for each defect through the thermal data processing perform. A comparison of phase and amplitude image defect detectability for each frequency, and the relationship between defect size and depth was developed. This result provides valuable information on aluminum material inspection.
\end{abstract}

